\title{
Factors Predicting Outcome of Total Thyroidectomy in Young Patients with Multiple Endocrine Neoplasia Type 2: A Nationwide Long-Term Follow-up Study
}

\author{
Jennifer M. J. Schreinemakers • Menno R. Vriens • Gerlof D. Valk • \\ Jan-Willem B. de Groot $\cdot$ John T. Plukker $\cdot$ Klaas (N.) M. A. Bax • \\ Jaap F. Hamming • Rob B. van der Luijt • Daniel C. Aronson • \\ Inne H. M. Borel Rinkes
}

Published online: 9 January 2010

(c) The Author(s) 2010. This article is published with open access at Springerlink.com

\begin{abstract}
Background Multiple endocrine neoplasia type 2 (MEN 2) is caused by a RET mutation in chromosome 10. All MEN 2 patients develop medullary thyroid carcinoma (MTC). The age-related risk of MTC is associated with the type of RET mutation. Our aim was to identify prognostic factors associated with recurrent MTC in MEN 2 patients.

Methods In a nationwide case-control study, all patients who underwent total thyroidectomy in the Netherlands under the age of 20 years were classified into standard (1), high (2), or very high risk (3) for MTC based on RETmutation type. Disease-free patients were compared with those with recurrent disease.

Results A total of 93 patients were included in the study. Sixty-six percent had MTC on histology, the youngest
\end{abstract}

J. M. J. Schreinemakers · M. R. Vriens .

I. H. M. Borel Rinkes ( $\square)$

Department of Surgery, University Medical Center Utrecht,

Hpnr. G04.228, Heidelberglaan 100, 3584CX Utrecht,

The Netherlands

e-mail: i.h.m.borelrinkes@umcutrecht.nl

\section{C. Aronson}

Pediatric Surgical Center of Amsterdam, Emma Children's Hospital AMC, Academic Medical Center and VU Medical Center, P.O. Box 22660, 1100 DD Amsterdam,

The Netherlands

G. D. Valk

Department of Endocrinology, University Medical Center Utrecht, Hpnr. G04.228, Heidelberglaan 100,

3584CX Utrecht, The Netherlands

J.-W. B. de Groot

Department of Endocrinology, University Medical Center

Groningen, P.O. Box 30.001, 9700 RB Groningen,

The Netherlands being 1 year old. Codon 634 was most affected. Sixteen (18\%) patients had persistent or recurrent disease, one of whom died. Significantly associated determinants of outcome in univariate analysis were higher age at surgery, no age-appropriate prophylactic surgery according to risk level, elevated preoperative calcitonin levels, affected codon, and the presence of lymph node metastases at surgery. On multivariate analysis only age of surgery was the single independent factor associated with persistent disease.

Conclusions Prophylactic thyroidectomy beyond the recommended age is associated with persistent/recurrent disease. In addition, codon 634 mutation is associated with a high risk of recurrence requiring early surgery for all these patients.

\section{J. T. Plukker}

Department of Surgery, University Medical Center Groningen, P.O. Box 30.001, 9700 RB Groningen, The Netherlands

K. (N.) M. A. Bax

Department of Pediatric Surgery, Erasmus Medical Center Rotterdam, P.O. Box 2060, 3000CB Rotterdam, The Netherlands

J. F. Hamming

Department of Surgery, Leiden University Medical Center, P.O. Box 9600, 2300RC Leiden, The Netherlands

\section{R. B. van der Luijt}

Department of Medical Genetics, University Medical Center Utrecht, Hpnr. G04.228, Heidelberglaan 100, 3584CX Utrecht, The Netherlands

Present Address:

D. C. Aronson

Department of Surgery/Pediatric Surgery, Radboud University Nijmegen Medical Center, P.O. Box 9101, 6500HB Nijmegen, The Netherlands 


\section{Introduction}

Multiple endocrine neoplasia type 2 (MEN 2) syndrome is a rare autosomal inherited complex of endocrine tumors caused by a mutation in the REarranged during Transfection (RET) proto-oncogene located on chromosome 10. According to the mutation type, MEN 2 can be divided into MEN type 2A and 2B. Almost all MEN 2A and 2B patients develop medullary thyroid carcinoma (MTC). MTC is a neuroendocrine cancer of the calcitonin-producing parafollicular C cells. In MEN 2A, pheochromocytomas and primary hyperparathyroidism can also present in, respectively, 40 and 20-30\% [1, 2]. In MEN 2B, primary hyperparathyroidism is absent; however, this disease is also characterized by the development of mucosal neuromas, intestinal ganglioneuromas, and, in some instances, a Marfanoid habitus. Familial medullary thyroid carcinoma (FMTC) is an inherited disorder caused by RET leading only to MTC. The progression from C-cell hyperplasia, the precursor lesion of MTC, to intrathyroidal MTC and eventually lymphatic and distant metastatic spread is age related [3-5].

In 1993 and 1994, the relationship between germline $R E T$ proto-oncogene mutations and MEN type 2A and type $2 \mathrm{~B}$ syndrome became apparent [6-8]. Since then, DNA analysis has become available to identify patients with hereditary MTC and to perform predictive testing in asymptomatic family members of mutation carriers. A genotype-phenotype relationship exists for different RET proto-oncogene mutations [9, 10]. The expression of MTC in terms of aggressiveness varies between the different codon mutations. Based on this genotype-phenotype relationship, patients are classified into different risk levels [11].

In earlier years, patients underwent surgery when they presented with a clinically apparent thyroid nodule or had elevated plasma calcitonin levels. By then, many had already progressed into late-stage MTC with lymph node or distant metastases. The availability of presymptomatic DNA analysis by direct detection of germline RET protooncogene mutations provided a rationale to perform prophylactic surgery because in $95 \%$ of patients with MEN 2 and FMTC, C-cell hyperplasia develops into MTC [4, 9, $10,12,13]$.

In recent years, timing of prophylactic thyroidectomy in asymptomatic carriers of germline RET gene mutations has been customized based on the age and type of mutation [9, 10]. In an effort to further optimize the management of these children, we conducted a nationwide study, including all Dutch patients who underwent total thyroidectomy in childhood in the past 25 years. We set out to study the differences in treatment for patients operated on at the age recommended by the current guidelines and those operated on at an older age. The second purpose of this study was to assess any differences in treatment and outcome in patients treated in the era before and after genetic testing became available. Finally, our aim was to analyze prognostic risk factors for recurrent MTC in these patients.

\section{Materials and methods}

This case control study was performed in the cohort of all Dutch patients with a germline RET mutation who were operated on in one of the eight university hospitals in the Netherlands from 1980 up until 2006. Inclusion criteria were MEN 2 syndrome, 19 years or younger at the time of surgery, and had undergone a total thyroidectomy.

The participating centers were the Free University Medical Center Amsterdam $(n=4)$, Leiden University Medical Center $(n=5)$, Academic Hospital Maastricht $(n=8)$, Radboud University Medical Center Nijmegen $(n=8)$, Academic Medical Center Amsterdam $(n=9)$, Erasmus Medical Center Rotterdam $(n=12)$, University Medical Center Groningen $(n=19)$, and University Medical Center Utrecht $(n=28)$.

In total, 93 patients were identified and data were collected retrospectively from hospital records in the participating centers by two authors (JMJS and MRV). Patients were divided into those who were operated on up to 1994 when predictive genetic testing became available ("pre$R E T$ era") and those after 1994 ("RET era"). The followup of the patients included in this study was part of regular medical care.

The approaches described in this article did not involve any randomization, experimental intervention, or questionnaire, and the anonymity of patients was not breached. Therefore, this article meets the conditions required under Dutch law (WGBO) for making medical and/or personal data available for statistical or other scientific research.

\section{Surgery}

Almost all 93 patients were operated on by a team consisting of a pediatric surgeon and an endocrine surgeon. All patients underwent total thyroidectomy. An additional prophylactic lymph node dissection of the central zone of the neck was not performed routinely. A central or lateral neck dissection was performed in case a suspicious lymph node was present during the surgery or on preoperative imaging. In the "pre-RET" era (until 1994), patients were operated on based on family history and/or elevated basal and/or stimulated calcitonin levels. In the "RET era" (after 1994), most patients were operated on based on the mutation analysis. Whenever possible, the parathyroid glands were left in situ. 


\section{Laboratory tests}

Calcitonin and pentagastrin-stimulated calcitonin levels were measured to determine the level of tumor activity. We could not retrieve all calcitonin levels, especially from the pre-RET era. However, it was often noted whether calcitonin levels were elevated. Furthermore, as each center used its own test methodology with according reference values (which differed between the participating hospitals and changed during the study period), we indicated whether these levels were elevated according to the local reference values [14]. Patients were considered cured when postoperative (pentagastrin-stimulated) calcitonin was in the lower limits of the reference values.

\section{Genetic testing}

In the Netherlands, screening for mutations in a RET proto-oncogene is carried out in one central genetics laboratory at the University Medical Center Utrecht. Mutation analysis was performed by PCR amplification followed by direct sequence analysis of exons 10, 11, 14, 13,15 , and 16 of the RET proto-oncogene. Sporadic cases of MTC were identified by mutation analysis of RET exons 15 and 16 in DNA isolated from tumor samples, following a normal $R E T$ mutation screening result on DNA from peripheral blood. All results of RET mutation screening were confirmed on a duplicate DNA sample. (PCR conditions and oligonucleotide primer sequences are available upon request from RBL.) Based on the affected codon, patients were stratified into three levels of risk of developing MTC according to the 2001 guidelines [11]: standard risk (level 1; codons 768, 790, 791, 804, and 891), high risk (level 2; codons 630, 634, 609, 611, 618, and 620), and very high risk (level 3; codons 918, 883, and 922) (Table 1) [15].

Pathological examination and tumor staging

Pathological examination of the thyroid specimens was performed separately at each hospital. In general, the thyroid specimen was fixed and transversally laminated in thin sections, which were then prepared for light microscopy.
The transition zone between the upper and the middle third of the thyroid lobes was the focus of attention, as $\mathrm{C}$ cells are normally situated in this area. The sections were investigated for the presence of $\mathrm{C}$ cells; characterization of cytoplasmic levels of calcitonin, chromogranin, and carcinoembryonic antigen (CEA); destructions of follicles; and amyloidal formation and invasion of vessels and or nerves by these cells. C-cell hyperplasia was diagnosed when more than $7 \mathrm{C}$ cells per follicle were present, there were clusters of $\mathrm{C}$ cells, or more than $20 \mathrm{C}$ cells were observed per field. MTC was diagnosed in case of atypia of $\mathrm{C}$ cells and their nuclei in combination with follicular basal membrane destruction as a sign of infiltrative C-cell proliferation [5].

\section{Statistical analysis}

Statistical analysis was performed using SPSS 16 (SPSS Inc., Chicago, IL). In this case control study, children with persistent or recurrent MTC were identified. Their exposure to possible prognostic factors was compared with those who were cured. Patients with C-cell hyperplasia were excluded from the analysis. Associations between categorical variables were evaluated with univariate analysis by $\chi^{2}$ analysis, and the in case of a sample size of 5 or fewer, by the Fischer' s exact test. Student's $t$ test was used to analyze differences in mean values, the Mann-Whitney $U$ test was used for differences in median values, and the ANOVA test was used for comparison of multiple groups. The correlation between factors was studied. A binary logistic regression analysis was performed to determine factors associated with recurrent disease. A $p$ value of less than 0.05 was considered to be significant. Factors included in the multivariate analysis were age at surgery, the affected codon, the risk level 1, 2, or 3, presence of lymph node metastasis, and preoperative calcitonin levels. Furthermore, for each patient it was determined whether surgery had been performed at an appropriate age as recommended by the guidelines (age-appropriate prophylactic surgery) and if the surgery had been performed in the pre-RET era (before 1994) or in the RET era (after 1994) [11]. This factor was included in the analysis as well.

Table 1 Risk level for MTC based on codon mutation

\begin{tabular}{llll}
\hline & Risk level & Codon & Recommended age of surgery \\
\hline 1 & Standard risk & $768,790,791,804$, and 891 & $5-10$ years \\
2 & High risk & $630,634,609,611,618$, and 620 & $<5$ years \\
3 & Very high risk & 918,883, and 922 & $<6$ months
\end{tabular}

Risk level of MTC based on the 1999 consensus statement from the Seventh International Workshop on Multiple Endocrine Neoplasia [11, 15] 


\section{Results}

Of the 93 patients identified, $38(41 \%)$ were male and 55 (59\%) female. Demographic, surgical, and follow-up data are summarized in Table 2.

The majority of cases had MEN 2A syndrome $(n=87$, $94 \%)$. The remaining patients had MEN 2B syndrome $(n=6,6 \%)$. Mutation analysis revealed that codon 634 (62 patients) was most frequently affected, followed by codon 618 (10 patients), codon 611 (9 patients), codon 918 (6 patients), codon 620 (3 patients), and codon 804 (1 patient). In two patients, who underwent surgery in the early 1980s, the genetic mutation could not be retrieved from the national database. In Table 2, patient characteristics are also given for patients operated on in the pre-RET era and in the RET era.

The median age of all patients at surgery was 8.9 years (IQR = 5.7-12.25). Patients who were operated on in the pre-RET era were older than patients operated in the RET era (10.6 vs. 7.8 years). A preoperatively elevated calcitonin level was found in 43 patients (46\%). A central compartment neck dissection was performed in 26 patients $(28 \%)$ and a modified lateral neck dissection in 8 $(9 \%)$, in most cases because of evident intraoperative lymph node metastases (Table 2). Seven patients (7\%) developed definitive hypoparathyroidism (15\% of patients in the pre-RET era and $4 \%$ in RET era) and two (2\%) patients suffered from permanent laryngeal nerve damage (none in the RET era). No association between the age at surgery, a central neck dissection, or the complication rate was found. None of the patients operated on who were younger than 5 years $(n=14)$ developed a complication. Five patients were not operated on prophylactically because four already had a clinically apparent tumor and another a palpable lymph node at the time of surgical intervention.

Univariate analysis showed that a higher age at surgery, no age-appropriate prophylactic surgery, affected codon and risk level, preoperative calcitonin level, and lymph node metastasis at operation were associated significantly with persistent or recurrent disease (Table 3). No association was seen with gender and different variants of codon 634 mutations. In multivariate analysis, only surgery at an older age was significantly associated with persistent or recurrent disease. In both the pre-RET era and the RET era, the majority of patients were not operated on at an ageappropriate age, but the percentage was significantly higher in the pre-RET era (96 vs. $69 \%, p=0.004$ ).

On histological examination 61 patients had already developed MTC (66\%), whereas $30(32 \%)$ patients displayed C-cell hyperplasia. Histological examination showed no abnormalities in only two patients. One of these patients had a Cys634Gly mutation and was operated on at the age of 1 year. The other had a Cys620Ser mutation and was 5 years of age at the time of surgery. There was no significant difference in median age between patients with C-cell hyperplasia and MTC (8.0 vs. 9.7 years). Lymph node metastases were observed in eight patients and were found only in patients with the Met918Thr mutation associated with MEN 2B syndrome and the Cys634Arg mutation, which is associated with MEN 2A syndrome. The youngest patient with lymph node metastases was 7 years old and had a Cys634Arg mutation. The median age at surgery of patients with histological lymph node metastases was 13 years $(\mathrm{IQR}=10-14)$. We found more lymph node metastases in patients operated on in the pre$R E T$ era than in the RET era (Table 2). None of the patients who had lymph node metastases had a normal preoperative calcitonin level and none had calcitonin levels lower than $40 \mathrm{pg} / \mathrm{ml}$. An overview of the earliest age at which MTC was demonstrated per codon in our series compared to literature is given in Table 4.

\section{Follow-up}

The median follow-up period was 7 years (IQR $=3-11$ ). At the time of the last visit, $75(81 \%)$ patients were diseasefree according to the postoperative calcitonin levels. The duration of follow-up was significantly different between patients who had persistent or recurrent disease and those who were cured. Thirteen of the 16 patients with persistent or recurrent disease at follow-up had elevated basal calcitonin or pentagastrin-stimulated calcitonin levels preoperatively. None of these 16 patients had been operated on at an appropriate age recommended by the guidelines. Three of the 27 patients who had MTC and normal preoperative calcitonin levels developed persistent or recurrent disease (Table 3). All patients with lymph node metastases at operation developed persistent or recurrent disease. All eight patients underwent a central neck dissection and five of them a lateral neck dissection as well. One MEN 2B patient died at the age of 18 (i.e., 6 years postoperatively) because of metastatic disease. No differences in presentation could be found between the different codon 634 mutations in terms of age at surgery and recurrent disease.

If we excluded patients who were operated on in the preRET era, patients who had persistent or recurrent disease were still older than those who were cured (12.3 vs. 7.9 years, $p=0.01$ ) and were less frequently operated on at an appropriate age $(p=0.034)$. These patients more often had an elevated preoperative calcitonin level $(p=0.04)$ and lymph node metastasis $(p=0.001)$ compared to those who were cured. The duration of follow-up, however, was not statistically different for both groups after excluding patients from the pre-RET era (8.2 vs. 6.2 years). 
Table 2 Patient characteristics

\begin{tabular}{|c|c|c|c|c|}
\hline & Overall & Pre-RET era $(n=25)(27 \%)$ & $R E T$ era $(n=68)(73 \%)$ & $p$ value $^{\mathrm{a}}$ \\
\hline Male:Female $[n(\%)]$ & $38(41 \%): 55(59 \%)$ & & & \\
\hline Median age (years) (IQR) & $8.9(5.7-12.6)$ & 10.6 years $(7.4-13.2)$ & $7.8(5.3-12.2)$ & 0.022 \\
\hline Median follow-up (years) (IQR) & $7(3-11)$ & & & \\
\hline \multicolumn{5}{|l|}{ Codon $[n(\%)]$} \\
\hline 634 & $62(67 \%)$ & & & \\
\hline Cys $\rightarrow$ Arg & 37 & & & \\
\hline Cys $\rightarrow$ Gly & 9 & & & \\
\hline Cys $\rightarrow$ Tyr & 8 & & & \\
\hline Cys $\rightarrow$ Trp & 8 & & & \\
\hline 620 & $3(3 \%)$ & & & \\
\hline Cys $\rightarrow$ Arg & 3 & & & \\
\hline 611 & $9(10 \%)$ & & & \\
\hline Cys $\rightarrow$ Tyr & 9 & & & \\
\hline 618 & $10(11 \%)$ & & & \\
\hline Cys $\rightarrow$ Ser & 8 & & & \\
\hline Cys $\rightarrow$ Tyr & 1 & & & \\
\hline Cys $\rightarrow$ Arg & 1 & & & \\
\hline 918 & $6(6 \%)$ & & & \\
\hline Met $\rightarrow$ Thr & 6 & & & \\
\hline $804 \mathrm{Val} \rightarrow$ Leu & $1(1 \%)$ & & & \\
\hline Unknown & $2(2 \%)$ & & & \\
\hline \multicolumn{5}{|l|}{ Risk level $(n)$} \\
\hline 1 (standard risk) & 1 & & & \\
\hline 2 (high risk) & 84 & & & \\
\hline 3 (very high risk) & 6 & & & \\
\hline Unknown & 2 & & & \\
\hline Preoperative calcitonin levels ${ }^{\mathrm{b}}$ & & & & ns \\
\hline Normal & $47(51 \%)$ & $14^{\mathrm{c}}$ & 33 & \\
\hline Elevated & $43(46 \%)$ & 10 & 33 & \\
\hline Unknown & $3(3 \%)$ & & & \\
\hline Age-appropriate surgery & & & & 0.004 \\
\hline Yes & $22(24 \%)$ & 1 & 21 & \\
\hline No & $71(76 \%)$ & 24 & 47 & \\
\hline \multicolumn{5}{|l|}{ Histology } \\
\hline C-cell hyperplasia & $30(32 \%)$ & 7 & 22 & ns \\
\hline Medullary thyroid carcinoma & $61(66 \%)$ & 18 & 43 & \\
\hline Normal histology & $2(2 \%)$ & & 2 & \\
\hline Lymph node metastasis & $8(9 \%)$ & 5 & 3 & 0.02 \\
\hline \multicolumn{5}{|l|}{ Disease status at follow-up } \\
\hline Disease free & $75(81 \%)$ & & & \\
\hline Recurrent disease & $16(17 \%)$ & & & \\
\hline Disease-related death & 1 & & & \\
\hline Unknown & 2 & & & \\
\hline
\end{tabular}

$n s$ not significant

${ }^{\text {a }}$ Comparison for pre-RET era and RET era

$\mathrm{b}$ The mean age of patients with normal calcitonin levels was 8.8 years versus a mean age of 9.0 years for patients with elevated calcitonin levels (not significantly different)

${ }^{c}$ Eleven of the 14 patients with normal calcitonin levels in the pre-RET era were operated on in the transition period between 1993 and 1994. Genetic testing had become available in the UMCU at that time. Two of the other three patients had C-cell hyperplasia 
Table 3 Factors associated with residual or recurrent disease in patients with MTC; univariate analysis

\begin{tabular}{|c|c|c|c|}
\hline Factor & $\begin{array}{l}\text { Residual/recurrent disease } \\
(n=16,27 \%)(n, \%)\end{array}$ & $\begin{array}{l}\text { No evidence of residual/recurrent } \\
\text { disease }(n=44,73 \%)(n, \%)\end{array}$ & Likelihood-ratio, $p$ value \\
\hline $\begin{array}{l}\text { Preoperative calcitonin levels } \\
\text { elevated }\end{array}$ & $N=13(81 \%)$ & $N=20(46 \%)$ & $6.5, p=0.011$ \\
\hline Codon $^{\mathrm{a}}$ & $\begin{array}{l}634, n=11 \\
918, n=4 \\
\text { Unknown, } n=1\end{array}$ & $\begin{array}{l}634, n=35 \\
618, n=3 \\
620, n=1 \\
611, n=3 \\
918, n=1 \\
\text { Unknown, } n=1\end{array}$ & $10.7, p=0.03$ \\
\hline Risk level $^{\mathrm{b}}$ & & & $7.1, p=0.007$ \\
\hline 1 & $n=0$ & $n=0$ & \\
\hline 2 & $n=11$ & $n=42$ & \\
\hline 3 & $n=4$ & $n=1$ & \\
\hline Unknown & $n=1$ & $n=1$ & \\
\hline $\begin{array}{l}\text { Lymph node metastases at total } \\
\text { thyroidectomy, total } n=8\end{array}$ & Lymph nodes present, $n=8$ & No lymph node metastases, $n=44$ & $26.0, p<0.0001$ \\
\hline $\begin{array}{l}\text { Surgery at an appropriate age } \\
\text { based on risk level }\end{array}$ & $\begin{array}{l}\text { No, } n=16 \\
\text { Yes, } n=0\end{array}$ & $\begin{array}{l}\text { No, } n=31 \\
\text { Yes, } n=13\end{array}$ & $9.3, p=0.002$ \\
\hline $\begin{array}{l}\text { Surgery in the pre- } R E T \text { era or } \\
\text { in the } R E T \text { era }\end{array}$ & $\begin{array}{l}\text { Pre-RET, } n=7 \\
R E T \text { era, } n=9\end{array}$ & $\begin{array}{l}\text { Pre-RET, } n=11 \\
R E T \text { era, } n=33\end{array}$ & $\begin{array}{l}\text { No significant difference } \\
p=0.14 \\
t \text { test or Mann-Whitney } U \text { test, } \\
p \text { value, and odds ratio }\end{array}$ \\
\hline Mean age at surgery ${ }^{c}$ (years) (range) & $12.1($ range $=1-19)$ & $8.6($ range $=1-19)$ & $p=0.002,95 \% \mathrm{CI}=1.2-5.7$ \\
\hline Mean follow-up (years) $( \pm S D)^{d}$ & $12.3( \pm 7.1)$ & $7.4( \pm 4.7)$ & $p=0.004,95 \% \mathrm{CI}=1.6-8.0$ \\
\hline
\end{tabular}

$C I$ confidence interval

${ }^{\text {a }}$ We calculated the percentage of patients who had MTC who were cured per codon. Seventy-six percent of patients with codon $634,100 \%$ of patients with a 618,620 , and 611 mutation, and only $20 \%$ of those with a 918 mutation were cured

b When we analyzed this for risk level, $79 \%$ of the patients with risk level 2 and $20 \%$ with risk level 3 were cured

${ }^{c}$ Single factor also significantly associated in multivariate analysis

d The duration of follow-up, however, was not statistically different between both groups after excluding patients from the pre-RET era (8.2 vs. 6.2 years)

\section{Discussion}

Due to the well-organized infrastructure of the relatively small country of the Netherlands, we were able to establish a unique nationwide overview of all Dutch patients with hereditary MTC who underwent a total thyroidectomy based on their RET proto-oncogene mutation since 1980. In the Netherlands all patients with MTC, especially young patients, are operated on in a university hospital. We included in our study all patients who underwent surgery in those hospitals and it is therefore unlikely that our results are biased because of selection of the population.

We evaluated potential prognostic factors responsible for the outcome of prophylactic thyroidectomy in MEN 2 patients. The presence of lymph node metastasis, elevated preoperative calcitonin levels, codon/risk level, no ageappropriate prophylactic surgery, and a higher age at surgery were associated with persistent/recurrent disease in univariate analysis. Higher age at surgery was the only independent prognostic variable for persistent or recurrent disease in multivariate analysis. This could be an indication that having the operation at an earlier age might lead to a better outcome, thus preventing recurrent disease. It appears that progression from C-cell hyperplasia into MTC is age-related [4, 5, 9]. Twenty-three percent of the patients who were operated on at a higher age than is currently recommended by the guidelines developed recurrent disease. These data, once again underline the importance of early, prophylactic surgery. However, the majority of patients who had C-cell hyperplasia were not operated on at the recommended age either. Based on the latter observation the argument could also be reversed because many patients are cured despite the fact that they are operated on at an older age than is recommended. We do, however, 
Table 4 Earliest presentation of medullary thyroid carcinoma in patients with a $R E T$ proto-oncogene mutation

\begin{tabular}{|c|c|c|c|c|}
\hline \multirow{2}{*}{$\begin{array}{l}\text { Codon with } R E T \\
\text { mutation and risk level }\end{array}$} & \multicolumn{2}{|c|}{ Earliest age at presentation of MTC (years) } & \multirow{2}{*}{$\begin{array}{l}\text { Earliest age of LN } \\
\text { metastases in current } \\
\text { series (years) }\end{array}$} & \multirow{2}{*}{$\begin{array}{l}\text { Earliest age at presentation } \\
\text { of MTC with LN metastases } \\
\text { (years) (Ref.) }\end{array}$} \\
\hline & Current series & (Ref.) & & \\
\hline 918 RL 3 & 1.13 & 0.75 [9] & 11 & $2.7[15]$ \\
\hline 634 RL 2 & 1.25 & $1.1[15]$ & 7 & $5[15]$ \\
\hline 611 RL 2 & 6 & $3[9]$ & - & $28[21]$ \\
\hline 620 RL 2 & 6 & $5[10]$ & - & $22[15]$ \\
\hline 618 RL 2 & 12 & 7 [9] & - & $11[10,22,23]$ \\
\hline 804 RL 1 & - & 6 [24] & - & 6 [24] \\
\hline
\end{tabular}

$M T C$ medullary thyroid carcinoma, $L N$ lymph node metastasis; -, not observed, $R L$ risk level of MTC based on the 1999 consensus statement from the Seventh International Workshop on Multiple Endocrine Neoplasia [11, 15]

want to keep in mind that almost one-fourth of the patients with MTC operated on at an older age developed persistent or recurrent disease.

In our series, all patients with nodal metastases had persistent or recurrent disease. Lymph node metastases appeared only in patients who were operated on at a relatively high age. One patient with persistent disease (elevated calcitonin levels) was only 1 year old (codon 918 mutation). Therefore, we believe that prophylactic thyroidectomy should not be delayed until after the age recommended in literature.

Three risk levels have been identified in the literature. Patients in the highest-risk level (level 3: codons 918, 883, and 922) tend to have an aggressive presentation of MTC. In our cohort, as expected, these patients presented with MTC at an early age. Therefore, they are in need of a more aggressive approach [9, 15].

Persistent and recurrent disease occurred only in children with mutations in codons 918 and 634. Furthermore, we found MTC in a 1-year-old child with a codon 634 mutation. Since no differences in biological behavior in follow-up were observed between the different codon 634 mutations in our series, we feel that all patients with a codon 634 mutation should be managed in the same manner.

Having surgery at a younger age is believed to be associated with a higher complication rate but we found no evidence of this in our study. The complication rate is more or less comparable with other series, even in the very young [16]. In addition, in our study, performing a central neck dissection was not associated with a higher complication rate. More complications occurred in the pre-RET era group. Although the numbers are too small to draw any firm conclusions, we think this difference between the complication rates of the pre-RET era and the RET era may was a result of the improvements in operative technique. Since endocrine surgeons are more experienced in thyroid surgery than pediatric surgeons, it is common practice in the Netherlands to perform surgery together to decrease the complication risk.

The youngest patient in our series with lymph node metastasis was 7 years old and carried a codon 634 mutation. Another report describes a 5-year-old patient with a nodal metastasis [17]. This is in contrast with two other large series in which lymph node metastases in patients younger than 11 and 14 years were not observed $[9,10]$. A central neck dissection is controversial. We do not think it is necessary to perform a routine prophylactic central neck dissection on patients younger than 5 years or if preoperative calcitonin levels are normal in patients who do not have MEN 2B syndrome. None of our patients with lymph node metastases had a normal preoperative elevated calcitonin. Machens [4] has suggested that a central neck dissection be performed if preoperative calcitonin levels are elevated, regardless of age.

As mentioned above, one MEN 2B (codon 918 mutation) patient with MTC and persistent disease after surgery was only 1 year old. In previous reports, MTC was reported in a patient with this mutation within the first 6 months of life. Furthermore, persistent disease has been reported within the first year of life [18-20]. Therefore, the consensus guidelines [11] recommend a total thyroidectomy together with a central lymph node dissection before the age of 6 months in MEN 2B patients. Lymph node metastases have been found in a 2.7-year-old child [18]. The 1 year old (codon 918) who had persistent disease in our series did not undergo a central neck dissection. The one patient who died in our series had MEN 2B syndrome (Met918Tyr mutation) and had been operated on when 12 years old.

Children with a risk level 2 (codon 634, 611, 618, or 620 mutation) have a high risk of developing MTC. Some reports have suggested that patients with a 634 mutation may be regarded as a separate group with a higher risk of MTC $[9,15]$. When we regarded codon 634 as a separate group, no recurrences of MTC were found in risk group 2 
(codons 611, 618, and 620). However, the knowledge that elevated preoperative calcitonin levels and/or suspicion of lymph node involvement means an increased risk of recurrent disease supports the advice of the consensus meeting to perform surgery on patients younger than the age of 5 [11].

It is generally believed that children with a relatively low risk (risk level 1) of developing MTC because of a germline mutation at codon $609,768,790,791,804$, or 891 should undergo a total thyroidectomy as well. The biological behavior of MTC in this group is less aggressive, which is confirmed in our cohort. The only patient in this group did not have MTC. Controversy remains regarding the age at which surgery should be performed. While some believe that patients in this category should be operated on before the age of 5, others advocate surgery in this risk group before the age of 10 [11]. However, patient numbers in our series were too small to draw any conclusions.

Limitations of this study are its retrospective nature and its associated disadvantages such as missing data from laboratory studies, the RET mutation in two cases, and indications for central neck dissection. Furthermore, as expected we found that patients operated on in the pre-RET era were older than those in the RET era. A larger proportion of the patients from the pre-RET era were not operated on at an appropriate age and had more lymph node metastases. However, when we excluded patients from the pre-RET era, the same factors were still associated with persistent disease.

Prophylactic thyroidectomy at a younger age is associated with less recurrence or metastasized MTC in patients harboring a RET mutation. Recurrent disease was observed only in patients with the 634 and 918 codon mutations who did not undergo total thyroidectomy at an appropriate age. Therefore, it seems justifiable to operate on patients in the very-high-risk group at an early age, as is currently recommended. All patients with codon 634 mutations should be treated equally.

Acknowledgments This work was supported by the Dutch Cancer Society and the Michael van Vloten Fund of the Dutch Surgical Association. On behalf of the endocrine and pediatric surgery working groups, we acknowledge and thank the following persons and participating centers for their involvement in this nationwide study: D. C. Aronson: Emma Children's Hospital AMC, Academic Medical Center/Free University Medical Center, Amsterdam; J. Th. M. Plukker, Th. Links, A. H. Brouwers, R. Rassouli, T. Wiggers, R. Hofstra, and J. W. de Groot: University Medical Center Groningen; J. F. Hamming, B. A. Bonsing, and J. Kievit: Leiden University Medical Center; N. D. Bouvy: Academic Hospital Maastricht; M. Wijnen: Radboud University Medical Center Nijmegen; T. L. v.d. Hoonaard and N. M. A. Bax: Erasmus Medical Center Rotterdam; J. M. J. Schreinemakers, M. R. Vriens, I. H.M. Borel Rinkes, G. D. Valk, R. B. van der Luijt, J. K. Ploos van Amstel, and D. C. v.d. Zee: University Medical Center Utrecht.
Open Access This article is distributed under the terms of the Creative Commons Attribution Noncommercial License which permits any noncommercial use, distribution, and reproduction in any medium, provided the original author(s) and source are credited.

\section{References}

1. Kraimps JL, Duh QY, Demeure M et al (1992) Hyperparathyroidism in multiple endocrine neoplasia syndrome. Surgery 112(6):1080-1086 (discussion 1086-1088)

2. Raue F, Frank-Raue K, Grauer A (1994) Multiple endocrine neoplasia type 2 Clinical features and screening. Endocrinol Metab Clin North Am 23(1):137-156

3. Machens A, Holzhausen HJ, Thanh PN et al (2003) Malignant progression from C-cell hyperplasia to medullary thyroid carcinoma in 167 carriers of RET germline mutations. Surgery 134(3):425-431

4. Machens A, Ukkat J, Brauckhoff M et al (2005) Advances in the management of hereditary medullary thyroid cancer. J Intern Med 257(1):50-59

5. Wolfe HJ, Melvin KE, Cervi-Skinner SJ et al (1973) C-cell hyperplasia preceding medullary thyroid carcinoma. N Engl J Med 289(9):437-441

6. Donis-Keller H, Dou S, Chi D et al (1993) Mutations in the RET proto-oncogene are associated with MEN 2A and FMTC. Hum Mol Genet 2(7):851-856

7. Lips CJ, Landsvater RM, Hoppener JW et al (1994) Clinical screening as compared with DNA analysis in families with multiple endocrine neoplasia type 2A. N Engl J Med 331(13): 828-835

8. Mulligan LM, Kwok JB, Healey CS et al (1993) Germ-line mutations of the RET proto-oncogene in multiple endocrine neoplasia type 2A. Nature 363(6428):458-460

9. Machens A, Niccoli-Sire P, Hoegel J et al (2003) Early malignant progression of hereditary medullary thyroid cancer. N Engl J Med 349(16):1517-1525

10. Skinner MA, Moley JA, Dilley WG et al (2005) Prophylactic thyroidectomy in multiple endocrine neoplasia type $2 \mathrm{~A}$. N Engl J Med 353(11):1105-1113

11. Brandi ML, Gagel RF, Angeli A et al (2001) Guidelines for diagnosis and therapy of MEN type 1 and type 2. J Clin Endocrinol Metab 86(12):5658-5671

12. de Groot JW, Links TP, Rouwe CW et al (2006) Prophylactic thyroidectomy in children who are carriers of a multiple endocrine neoplasia type 2 mutation: description of 20 cases and recommendations based on the literature. Ned Tijdschr Geneeskd 150(6):311-318

13. Gagel RF, Tashjian AH Jr, Cummings T et al (1988) The clinical outcome of prospective screening for multiple endocrine neoplasia type 2a. An 18-year experience. N Engl J Med 318(8): 478-484

14. Orlandi F, Caraci P, Mussa A et al (2001) Treatment of medullary thyroid carcinoma: an update. Endocr Relat Cancer 8(2):135-147

15. Machens A, Dralle H (2007) Genotype-phenotype based surgical concept of hereditary medullary thyroid carcinoma. World J Surg 31(5):957-968

16. Dralle H, Gimm O, Simon D et al (1998) Prophylactic thyroidectomy in 75 children and adolescents with hereditary medullary thyroid carcinoma: German and Austrian experience. World J Surg 22(7):744-750 (discussion 750-741)

17. Gill JR, Reyes-Mugica M, Iyengar S et al (1996) Early presentation of metastatic medullary carcinoma in multiple endocrine 
neoplasia, type IIA: implications for therapy. J Pediatr 129(3): 459-464

18. Samaan NA, Draznin MB, Halpin RE et al (1991) Multiple endocrine syndrome type IIb in early childhood. Cancer 68(8): $1832-1834$

19. Skinner MA, DeBenedetti MK, Moley JF et al (1996) Medullary thyroid carcinoma in children with multiple endocrine neoplasia types 2A and 2B. J Pediatr Surg 31(1):177-181 (discussion 181-172)

20. Stjernholm MR, Freudenbourg JC, Mooney HS et al (1980) Medullary carcinoma of the thyroid before age 2 years. J Clin Endocrinol Metab 51(2):252-253

21. Siggelkow H, Melzer A, Nolte W et al (2001) Presentation of a kindred with familial medullary thyroid carcinoma and
Cys611Phe mutation of the RET proto-oncogene demonstrating low grade malignancy. Eur J Endocrinol 144(5):467-473

22. Lindskog S, Nilsson O, Jansson S et al (2004) Phenotypic expression of a family with multiple endocrine neoplasia type $2 \mathrm{~A}$ due to a RET mutation at codon 618. Br J Surg 91(6):713-718

23. Neocleous V, Passalaris T, Spanou E et al (2004) Description of the first two seemingly unrelated Greek Cypriot families with a common C618R RET proto-oncogene mutation. Genet Test 8(2):163-168

24. Frohnauer MK, Decker RA (2000) Update on the MEN 2A c804 RET mutation: is prophylactic thyroidectomy indicated? Surgery 128(6):1052-1057 (discussion 1057-1058) 